\title{
The Influence of Education Level on Tinnitus and Quality of Life in Korean Adults
}

\author{
Da Jung Jung, Myung Hoon Yoo, Yu Jin Jung, Hyun Ju Lee, and Kyu-Yup Lee ${ }^{\mathbb{D}}$ \\ Department of Otorhinolaryngology-Head and Neck Surgery, School of Medicine, Kyungpook National University Hospital, Daegu, Korea
}

\author{
이명 및 삶의 질에 관한 교육 수준의 영향 \\ 정다정 · 유명훈 · 정유진 · 이현주 · 이규엽 \\ 경북대학교 의과대학 이비인후-두경부외과학교실
}

Received November 9, 2018

Revised March 29, 2019

Accepted April 2, 2019

Address for correspondence

Kyu-Yup Lee, MD

Department of Otorhinolaryngology-

Head and Neck Surgery,

School of Medicine,

Kyungpook National University

Hospital, 130 Dongdeok-ro, Jung-gu,

Daegu 41944, Korea

Tel $+82-53-420-5785$

Fax $+82-53-423-4524$

E-mail kylee@knu.ac.kr
Background and Objectives We aimed to evaluate the effect of the level of education on the prevalence of tinnitus and quality of life (QoL) in the Korean population.

Subjects and Method Our study included 3155 participants from a representative sample. Tinnitus was measured using questionnaires that evaluated perception of ringing, buzzing, roaring, or hissing sounds. Participants were asked to indicate "Yes" or "No" to these questions. Participants who felt that the sensation was "A little annoying" or "Very annoying" were considered to have moderate or severe tinnitus, respectively. Hearing thresholds were measured using an automatic audiometer. QoL was evaluated using the EuroQoL (EQ) scale.

Results The number of participants in the low, middle, and high education groups were 1521,967 , and 667, respectively. Participants with any degree of tinnitus or moderate to severe tinnitus increased as the level of education decreased. Multivariate logistic regression analysis showed that the low education group had significantly higher odds ratios compared to the middle or high education groups for moderate to severe tinnitus. Abnormal EQ 5-dimensions (EQ-5D) were higher in participants with tinnitus and a low level of education. For participants, univariate and multivariate analyses showed EQ-5D index and EQ-visual analog scale in the low education group were lower than that in the other groups.

Conclusion Our study showed that low education level was associated with moderate to severe tinnitus in the Korean adult population. Participants with tinnitus and a low level of education had poorer QoL than those with tinnitus and a high level of education. Korean J Otorhinolaryngol-Head Neck Surg 2019;62(9):499-506

Key Words Education · Hearing loss - Quality of life · Tinnitus.

\section{Introduction}

Tinnitus is a well-known clinical condition with subjective perception of sound that is heard only be the person affected. Previous large scale studies have reported the prevalence rate of tinnitus as approximately $11.9-25.3 \%{ }^{1-3)}$ Previous studies suggest several factors, including otologic causes, sys-

This is an Open Access article distributed under the terms of the Creative Commons Attribution Non-Commercial License (https://creativecommons.org/licenses/by-nc/4.0) which permits unrestricted non-commercial use, distribution, and reproduction in any medium, provided the original work is properly cited. temic diseases, and psychological problems that could be associated with the development of tinnitus. ${ }^{4)}$ Tinnitus is associated with a decreased quality of life (QoL), especially if it is severe. $^{5-7)}$ Various modes of therapy, including medications, cognitive-behavioral therapy, and sound therapy have been tried, but their efficacy remains unclear. ${ }^{8)}$ Consequently, unresolved tinnitus leads to lack of improvement of QoL. Therefore, apart from treatment alone, identification of additional factors that lead to the development of tinnitus or a decrease in QoL may be helpful to understand the pathophysiology 
and aid in the management of patients with tinnitus.

Participants with a low education level have lower self-esteem, cognitive, and control capacities compared with those with a higher education level. ${ }^{7)}$ Regarding the association between tinnitus and psychologic factors, positive effects of education level on tinnitus or QoL are expected. However, not many studies have addressed the association between the level of education and prevalence of tinnitus, and the results have been inconclusive. Shargorodsky et al., in a study among the US population, showed no such association; However, Fujii et al., ${ }^{2)}$ in a Japanese study showed a lower odds ratio for tinnitus in men with a higher level of education. In addition, few studies have addressed the effect of education level on QoL in patients with tinnitus. We aimed to evaluate the effect of the level of education on the prevalence of tinnitus and QoL in the Korean population.

\section{Subjects and Method}

\section{Study population}

We conducted the study between 2010-2012 using data from the Korean National Health and Nutrition Examination Survey (KNHANES). This is a representative sample of a national, multi-stage, stratified survey of the Korean population. The sample included 13132 participants aged between 4080 years. We excluded 9583 participants due to missing clinical data. Trained otologists examined the both tympanic membrane of participants. We excluded subjects with abnormal findings on the tympanic membrane $(n=394)$. Thus, 3155 participants were included in the final analysis. None of the participants had a history of otitis media. Local Ethics Committee approval was obtained for our study. The board waived the need for informed consent because the subjects' records and information were anonymized and de-identified prior to the analysis (IRB No. KNUH 2017-11-005-001).

\section{Study variables}

We collected clinical, demographic and laboratory data including those regarding: age, sex, body mass index (BMI; kg/ $\mathrm{m}^{2}$ ), history of diabetes mellitus (DM), hypertension (HTN), smoking, alcohol consumption, cerebrovascular accident (CVA), dyslipidemia, depression, and stress related disorders. We also collected data on occupation, household incomes (1000 won/ month), average hearing thresholds (AHT), and QoL scores.

The education level was divided into low (less than high school), middle (high school), and high level of education (col- lege or more). DM was defined as fasting glucose level $\geq 126$ $\mathrm{mg} / \mathrm{dL}$, a self-reported history DM, or the use of anti-diabetic medication. HTN was defined as blood pressure $\geq 140 / 90$ $\mathrm{mm} \mathrm{Hg}$, a self-reported history of HTN, or the use of anti-hypertensive medication. Smokers were classified as current smokers, ex-smokers, or non-smokers. Non-smokers were defined as individuals who had smoked $<100$ cigarettes in their lifetime, ex-smokers were those who had smoked $\geq 100$ cigarettes in their lifetime and had ceased smoking $\geq 1$ year prior to the survey, current smokers were those who had smoked $\geq 100$ cigarettes in their lifetime or had ceased smoking $<1$ year prior to the survey. Alcohol consumption was classified according to the Korean definition of standard drinking, based on the World Health Organization classification, ${ }^{9)}$ intake into the 3 categories: abstinence (no alcohol consumption during the 12 months prior to the survey), moderate consumption (women, 0.1-19.99 $\mathrm{g}$ of pure alcohol/day; men, 0.1-39.99 $\mathrm{g}$ of pure alcohol/day), and heavy consumption (women, $\geq 20 \mathrm{~g}$ of pure alcohol/day; men, $\geq 40$ g of pure alcohol/day).

Cerebrovascular accident was defined as a self-reported history of ischemic or hemorrhagic stroke; Coronary artery disease was defined as a self-reported history of angina or myocardial infarction. Depression, osteoarthritis, rheumatoid arthritis, and dyslipidemia were defined based on self-reports. We classified occupation according to the intensity of physical activity involved into three groups: non-labor intensive (manager, expert, specialist, clerk, service worker, sales person, technician, mechanic, production worker, or engineer), labor intensive (farmer, fisherman, or laborer), or no-occupation group (unemployed). Stress level was evaluated based on self-assessment by the participant and classified as no stress, or mild, moderate, and severe stress.

\section{Survey of tinnitus and hearing thresholds}

We evaluated tinnitus using questionnaires that evaluated perception of ringing, buzzing, roaring, or hissing sound, as previously reported. ${ }^{3)}$ Participants were asked to indicate "Yes" or "No" to these questions. If the answer was "Yes," they were asked, "Do these sounds bother you?" and had to choose between "No," "A little annoying," or "Very annoying." Participants who felt that the sensation was "A little annoying" or "Very annoying" were considered to have moderate and severe tinnitus respectively. History of exposure to explosive and occupational noise was sought for and classified as positive or negative. An explosive noise was defined as a sudden loud noise, similar to that arising from and an explosion or a gun- 
shot. Exposure to occupational noise was considered positive if participants had worked in a location with loud machinery for $\geq 3$ months. Exposure to loud noise defined as the needed to raise the voice to carry out a conversation. Hearing thresholds were measured using an automatic audiometer at $0.5,1$, $2,3,4$, and $6 \mathrm{kHz}$. AHT was calculated as the pure-tone average of the thresholds at $0.5,1,2$, and $3 \mathrm{kHz}$, bilaterally. Hearing loss (HL) was defined as an AHT of $>25 \mathrm{~dB}$.

\section{Survey for QoL}

QoL was evaluated using the EuroQoL (EQ). ${ }^{10)}$ EQ consists of two parts including a health-status descriptive system (EQ 5-dimension, EQ-5D), and the EQ-visual analogue scale (EQVAS). EQ-5D consist of five dimension: mobility, self-care, usual activities, pain/discomfort, and anxiety/depression. For each dimension, participants chose between "No problem," "Some problems," or "Extreme problems" depending on their state of health. If participants responded "Some problems" or "Extreme problems" to any dimension, they were classified as abnormal. For EQ-VAS, participants answered the question "How is your state of health today?" on a scale ranging from 0 (worst health) to 100 (best health). The EQ$5 \mathrm{D}$ index was calculated using a combination of responses to EQ-5D and the scoring algorithm from the Korean value set. ${ }^{11)}$ Scores ranged from 0 (worst health, equivalent to death) to 1 (optimal health).

\section{Statistical analyses}

Data were analyzed using SPSS statistics software package (version 23; IBM Corp., Armonk, NY, USA). Chi-square test was used to analyze non-continuous data. One-way analysis of covariance, followed by a post-hoc Tukey comparison, or t-test was used to analyze continuous data. Logistic regression analysis was used to analyze the association between education level and tinnitus. Multivariate analysis was performed for logistic regression or analysis of covariance. Multivariate analysis was performed to adjust for age, sex, BMI, comorbidities [DM, HTN, CVA, coronary artery disease (CAD), osteoarthritis, rheumatoid arthritis, depression, and dyslipidemia], smoking, alcohol consumption, AHT, occupation, household income, exposure to occupational or explosive noise, and stress level. If the association between education level and QoL in participants with tinnitus was analyzed, the severity of tinnitus was added as a covariate.

Although multivariate analysis was performed, most baseline characteristics were different among groups. To adjust for differences in baseline characteristics between the low education group and middle or high education groups (collectively, non-low education group), we estimated propensity scores using logistic regression models using the following variables: age, sex, BMI, comorbidities (DM, HTN, CVA, CAD, osteoarthritis, rheumatoid arthritis, depression, and dyslipidemia), smoking, alcohol consumption, AHT, occupation, household income, exposure to occupational or explosive noise, and stress level. Participants in the low education group were matched with participants in the non-low education group using a 1:1 nearest neighbor matching without replacement and with a matching tolerance (caliper) of 0.2 ; the nearest neighborhood matching was based on propensity scores. A $p$-value of $<0.05$ was considered statistically significant.

\section{Results}

\section{Clinical characteristics}

The number of participants in the low, middle, and high education groups were 1521, 967, and 667, respectively (Table 1). Participants in the high education group were younger. AHT increased with a decrease in education level. Household income was higher in participants with a higher level of education. The prevalence of comorbidities (DM, HTN, CVA, CAD, osteoarthritis, rheumatoid arthritis, dyslipidemia, and depression) was lower with a higher level of education. The number of current smokers was higher in participants with higher level of education. In the low education level group, there were more participants who were involved in labor intensive occupation or had no occupation. Participants without stress were highest in the low education group among the three groups.

\section{Association between education level and tinnitus}

Fig. 1 shows the distribution of participants according to the presence or severity of tinnitus. The number of participants with any degree of tinnitus was $413(27.2 \%)$ in the low education group, $173(17.9 \%)$ in the middle education group, and $105(15.7 \%)$ in the high education group $(p<0.001)$. Those with moderate to severe tinnitus was $186(12.2 \%)$ in the low education group, $41(4.2 \%)$ in the middle education group, and $27(4.0 \%)$ in the high education group $(p<0.001)$. Participants with any degree of tinnitus or moderate to severe tinnitus increased as the level of education decreased. Logistic regression showed no significant correlation between tinnitus and education level (Table 2). However, multivariate lo- 
Table 1. Participants' characteristics according to education level

\begin{tabular}{|c|c|c|c|c|}
\hline Variables & $\begin{array}{l}\text { Low education group } \\
(n=1521)\end{array}$ & $\begin{array}{l}\text { Middle education group } \\
(n=967)\end{array}$ & $\begin{array}{l}\text { High education group } \\
(\mathrm{n}=667)\end{array}$ & $p$-value \\
\hline Age (years) & $62.4 \pm 9.0$ & $52.1 \pm 9.5^{*}$ & $49.8 \pm 9.5^{*+}$ & $<0.001$ \\
\hline Sex (men, \%) & $538(35.4)$ & $456(47.2)$ & $414(62.1)$ & $<0.001$ \\
\hline Body mass index $\left(\mathrm{kg} / \mathrm{m}^{2}\right)$ & $24.2 \pm 3.1$ & $23.8 \pm 3.0^{*}$ & $24.0 \pm 3.0$ & 0.008 \\
\hline Diabetes mellitus (\%) & $283(18.6)$ & $103(10.7)$ & $59(8.8)$ & $<0.001$ \\
\hline Hypertension (\%) & $768(50.5)$ & $314(32.5)$ & $184(27.6)$ & $<0.001$ \\
\hline Smoking habitus (\%) & & & & $<0.001$ \\
\hline Non-smoker & $974(64.0)$ & $545(56.4)$ & $3121(46.8)$ & \\
\hline Ex-smoker & $312(20.58)$ & $208(21.5)$ & $185(27.7)$ & \\
\hline Current smoker & $235(15.5)$ & $214(22.1)$ & $170(25.5)$ & \\
\hline Alcohol intake (\%) & & & & $<0.001$ \\
\hline Abstinence & $604(39.7)$ & $236(24.4)$ & $132(19.8)$ & \\
\hline Moderate intake & $849(55.8)$ & $680(70.3)$ & $506(75.9)$ & \\
\hline Heavy intake & $68(4.5)$ & $51(5.3)$ & $29(4.3)$ & \\
\hline Coronary artery disease (\%) & $73(4.8)$ & $25(2.6)$ & $16(2.4)$ & 0.003 \\
\hline Cerebrovascular accident (\%) & $40(2.6)$ & $16(1.7)$ & $4(0.6)$ & 0.005 \\
\hline Average hearing threshold (dB) & $25.7 \pm 15.7$ & $16.8 \pm 12.1^{*}$ & $14.3 \pm 10.2^{*+}$ & $<0.001$ \\
\hline Household income (1000 won/month) & $267.7 \pm 590.3$ & $448.1 \pm 707.8^{*}$ & $662.6 \pm 1428.9 *+$ & $<0.001$ \\
\hline Exposure to occupational noise (\%) & $253(16.6)$ & $153(15.8)$ & $42(6.3)$ & $<0.001$ \\
\hline Exposure to explosive noise (\%) & $271(17.8)$ & $195(20.2)$ & $184(27.6)$ & $<0.001$ \\
\hline \multicolumn{5}{|l|}{ Occupation (\%) } \\
\hline Non-labor intensive & $332(21.8)$ & $478(49.4)$ & $433(64.9)$ & \\
\hline Labor intensive & $514(33.8)$ & $151(15.6)$ & $32(4.8)$ & \\
\hline Non-occupation & $675(44.4)$ & $338(35.0)$ & $202(30.3)$ & \\
\hline Osteoarthritis (\%) & $377(24.8)$ & $68(7.0)$ & $25(3.7)$ & $<0.001$ \\
\hline Rheumatoid arthritis (\%) & $62(4.1)$ & $15(1.6)$ & $6(0.9)$ & $<0.001$ \\
\hline Dyslipidemia (\%) & $256(16.8)$ & $97(10.0)$ & $79(11.8)$ & $<0.001$ \\
\hline Depression (\%) & $89(5.9)$ & 31 (3.2) & $17(2.5)$ & $<0.001$ \\
\hline Stress (\%) & & & & $<0.001$ \\
\hline No & $332(21.8)$ & $136(14.1)$ & $83(12.4)$ & \\
\hline Some stress & 820 (53.9) & $616(63.7)$ & $411(61.6)$ & \\
\hline Moderate stress & $290(19.1)$ & $184(19.0)$ & $151(22.6)$ & \\
\hline Severe stress & $79(5.2)$ & $31(3.2)$ & $22(3.3)$ & \\
\hline
\end{tabular}

The data are expressed as counts (percentages) for categorical variables and as mean \pm standard deviations for continuous variables. $p$-values were tested by one-way analysis of variance, followed by a post-hoc Tukey comparison for continuous variables and $\chi^{2}$ test for categorical variables. $* p<0.05$ vs. the low education group, ${ }^{\dagger} p<0.05$ vs. the middle education group

gistic regression analysis showed that the low education group had significantly higher odds ratios of 1.62 [95\% confidence interval (CI): 1.01-2.61] compared to the high education group, and 1.78 (95\% CI: 1.20-2.64) compared to the middle education group for moderate to severe tinnitus.

\section{Association between education level and EQ-5D dimensions in participants with tinnitus}

The numbers of participants in the low, middle, and high education groups who experienced tinnitus was 413, 173, and 105 , respectively. Participants who had problems with mobil- ity included $153(37.0 \%)$ in the low education group, 21 (12.1\%) in the middle education group, and $6(5.7 \%)$ in the high education group ( $p<0.001)$. Regarding self-care, $42(10.2 \%)$ participants had problems in the low education group, 4 (2.3\%) in the middle education group, and $1(1.0 \%)$ in the high education group $(p<0.001) .106(25.7 \%)$ participants had problems with usual activities in the low education group, while $14(8.1 \%)$ in the middle education group, and $3(2.9 \%)$ in the high education group had problems $(p<0.001)$. Pain/discomfort was experienced by $180(43.6 \%)$ in the low education group, $35(20.2 \%)$ in the middle education group, and 22 
$(21.0 \%)$ in the high education group $(p<0.001)$. Anxiety/depression was experienced by $87(21.1 \%)$ in low education group, while $23(13.3 \%)$ in middle education group, and 10 $(9.5 \%)$ in the high education group experienced anxiety/depression ( $p=0.005$ ). Abnormal EQ-5D dimensions were higher in participants with a low level of education.

\section{Association between education level and EQ-VAS or EQ-5D index in participants with tinnitus}

For participants with tinnitus, EQ-5D indexes in the low, middle, and high education groups were $0.86 \pm 0.17$ [mean \pm standard deviation (SD)], $0.95 \pm 0.08$, and $0.97 \pm 0.06$, respectively $(p<0.001)$ (Fig. 2A). On multivariate analysis, EQ-5D

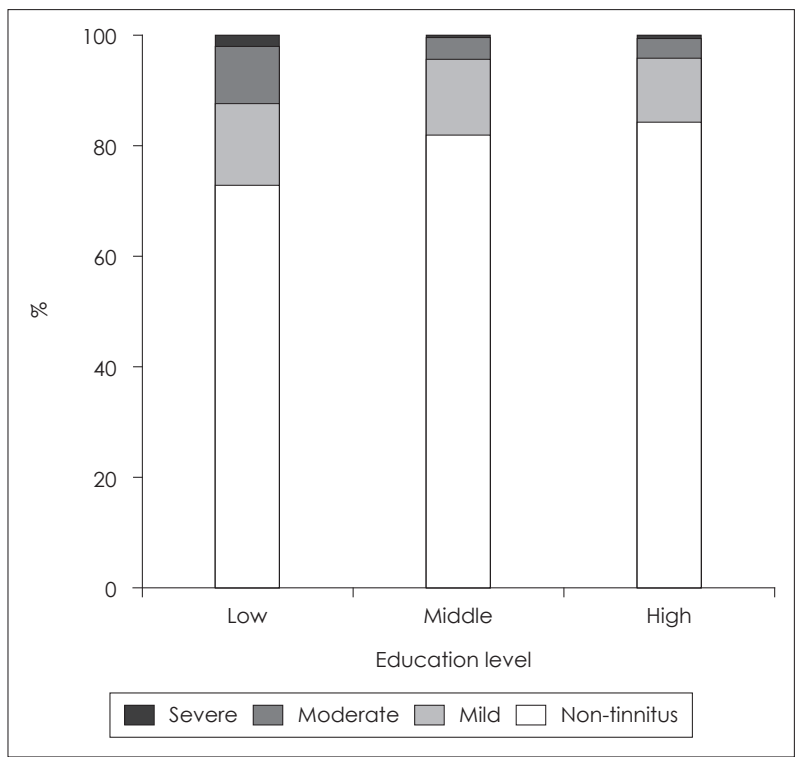

Fig. 1. The presence or severity of tinnitus according to education level. The number of participants without tinnitus, with mild, moderate, or severe tinnitus was $1180(72.8 \%), 227(14.9 \%), 160$ $(10.5 \%)$, and $26(1.7 \%)$ in the low education group, $794(82.1 \%)$, $132(13.7 \%), 37(3.8 \%)$, and $4(0.4 \%)$ in the middle education group, and $562(84.3 \%), 78(11.7 \%), 23(3.4 \%)$, and $4(0.6 \%)$ in the high education group $(p<0.001)$, respectively. indexes in the low, middle, and high education groups were $0.88 \pm 0.01$ [mean \pm standard error (SE)], $0.92 \pm 0.01$, and $0.94 \pm 0.01$, respectively ( $p=0.003$ ). EQ-VAS in the low, middle, and high education groups were $65.2 \pm 21.4$ (mean \pm SD), $72.7 \pm 17.5$, and $77.0 \pm 13.9$, respectively ( $p<0.001)$ (Fig. 2B). On multivariate analysis, EQ-VAS in the low, middle, and high education groups were $66.8 \pm 1.0$ (mean $\pm \mathrm{SE}$ ), 70.5 \pm 1.6 , and $74.3 \pm 2.1$, respectively $(p=0.010)$. On univariate and multivariate analyses, EQ-5D index and EQ-VAS in the low education group were lower than that in the other groups.

\section{Subgroup analysis according to $\mathrm{HL}$}

Participants with tinnitus were divided into two groups based on the presence of HL. 309 participants (239 in the low education group, 47 in the middle education, and 23 in the high education group) had HL, while 382 (174 in the low education group, 126 in the middle education group, and 82 in the high education group) did not. We analyzed the distribution of participants with abnormal EQ-5D dimensions (Table 3). Regardless of the presence of HL, most EQ-5D dimensions, EQ-5D index, and EQ-VAS showed similar trends, with lower QoL scores as education level decreased.

\section{Analysis using propensity matched cohorts}

For propensity analysis, we divided participants into low and non-low education groups with 782 matched pairs. The estimated distribution of propensity scores was similar after matching between low and non-low education groups (Supplementary Fig. 1). After matching, baseline characteristics except occupation were not significantly different between the two groups (Supplementary Table 1). The number of participants with any tinnitus was 177 (22.6\%) in the low education group and $156(19.9 \%)$ in the non-low education group $(p=0.195)$. Those with moderate to severe tinnitus was 70

Table 2. Logistic regression analyses of any tinnitus or moderate to severe tinnitus according to education level

\begin{tabular}{|c|c|c|c|c|c|c|c|c|}
\hline & \multicolumn{4}{|c|}{ Dependent variable: any tinnitus } & \multicolumn{4}{|c|}{ Dependent variable: moderate to severe tinnitus } \\
\hline & \multicolumn{2}{|c|}{ Univariate } & \multicolumn{2}{|c|}{ Multivariate* } & \multicolumn{2}{|c|}{ Univariate } & \multicolumn{2}{|c|}{ Multivariate* } \\
\hline & OR $(95 \% \mathrm{Cl})$ & p-value & OR $(95 \% \mathrm{Cl})$ & $p$-value & OR $(95 \% \mathrm{Cl})$ & p-value & OR $(95 \% \mathrm{Cl})$ & p-value \\
\hline High (ref) & - & - & - & - & - & - & - & - \\
\hline Middle & $1.17(0.89-1.52)$ & 0.256 & $1.02(0.77-1.35)$ & 0.887 & $1.05(0.64-1.72)$ & 0.849 & $0.88(0.53-1.47)$ & 0.621 \\
\hline Low & $2.00(1.57-2.53)$ & $<0.001$ & $1.15(0.87-1.53)$ & 0.322 & $3.30(2.18-5.00)$ & $<0.001$ & $1.62(1.01-2.61)$ & 0.046 \\
\hline Middle (ref) & - & - & - & - & - & - & - & - \\
\hline Low & $1.71(1.40-2.09)$ & $<0.001$ & $1.13(0.89-1.43)$ & 0.317 & $3.15(2.22-4.46)$ & $<0.001$ & $1.78(1.20-2.64)$ & 0.004 \\
\hline
\end{tabular}

* multivariate analysis was adjusted for age, sex, body mass index, diabetes mellitus, hypertension, cerebrovascular accident, coronary artery disease, osteoarthritis, rheumatoid arthritis, depression, dyslipidemia, smoking habit, alcohol intake, averaged hearing threshold, household income, exposure to occupational or explosive noise, occupation, and stress. OR: odds ratio, Cl: confidence interval, Low: low education, Middle: middle education, High: high education 

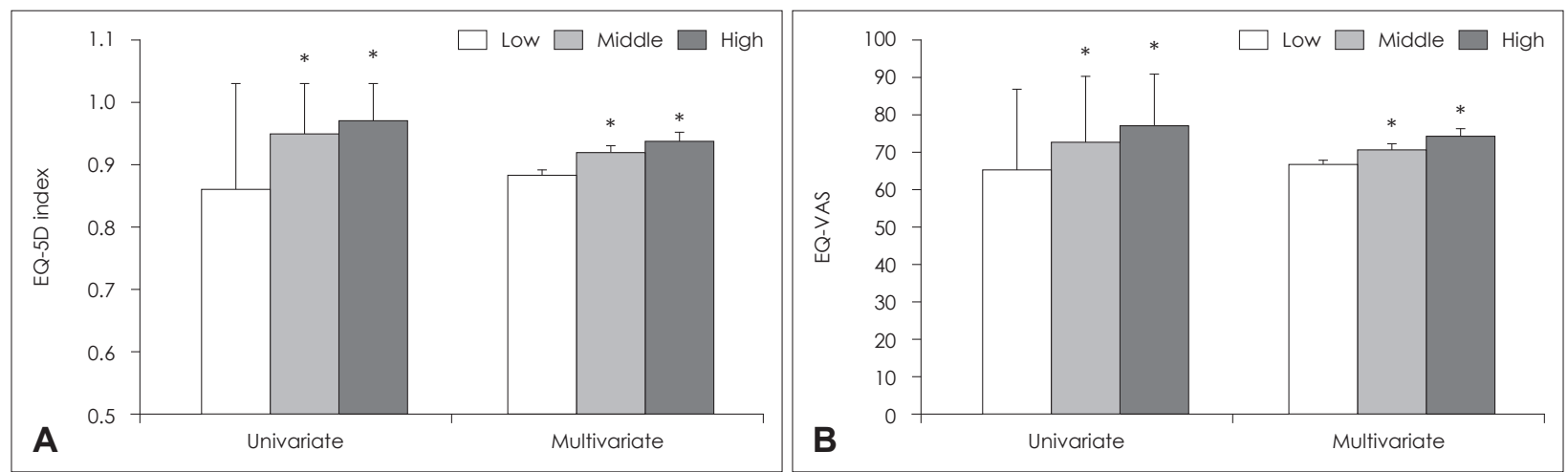

Fig. 2. EQ-5D index and EQ-VAS according to education level in participants with tinnitus (EQ-5D index; $A$, EQ-VAS; B). Data are expressed as mean and standard deviation for univariate analysis and mean and standard error for multivariate analysis. ${ }^{*} p<0.05$ vs. low education group. EQ-5D: EuroQoL 5-dimension, EQ-VAS: EuroQoL-visual analog scale.

Table 3. Comparison of quality of life scales according to education level and the presence of hearing loss in participants with tinnitus

\begin{tabular}{|c|c|c|c|c|c|c|c|c|}
\hline & \multicolumn{4}{|c|}{$\mathrm{HL}(+)$} & \multicolumn{4}{|c|}{$\mathrm{HL}(-)$} \\
\hline & Low & Middle & High & $p$-value & Low & Middle & High & $\mathrm{p}$-value \\
\hline Mobility (\%) & $94(39.3)$ & $10(21.3)$ & $1(4.3)$ & $<0.001$ & $59(33.9)$ & $11(8.7)$ & $5(6.1)$ & $<0.001$ \\
\hline Self-care (\%) & $31(13.0)$ & $3(6.4)$ & $1(4.3)$ & 0.234 & $11(6.3)$ & $1(0.8)$ & $0(0)$ & 0.005 \\
\hline Usual activities (\%) & $65(27.2)$ & $5(10.6)$ & $0(0)$ & 0.001 & $41(23.6)$ & $9(7.1)$ & $3(3.7)$ & $<0.001$ \\
\hline Pain/discomfort (\%) & $105(43.9)$ & $12(25.5)$ & $2(8.7)$ & 0.001 & $75(43.1)$ & $23(18.3)$ & $20(24.4)$ & $<0.001$ \\
\hline Anxiety/depression (\%) & $45(18.8)$ & $14(29.8)$ & $0(0)$ & 0.012 & $42(24.1)$ & $9(7.1)$ & $10(12.2)$ & $<0.001$ \\
\hline EQ-5D index & $0.85 \pm 0.18$ & $0.92 \pm 0.11^{*}$ & $0.98 \pm 0.05^{*}$ & $<0.001$ & $0.88 \pm 0.15$ & $0.96 \pm 0.07^{*}$ & $0.98 \pm 0.06^{*}$ & $<0.001$ \\
\hline EQ-VAS & $63.9 \pm 22.3$ & $71.4 \pm 16.8^{*}$ & $82.7 \pm 9.6^{*}$ & $<0.001$ & $66.9 \pm 20.1$ & $73.1 \pm 17.8^{*}$ & $75.4 \pm 14.6^{*}$ & 0.001 \\
\hline
\end{tabular}

The data are expressed as counts (percentages) for categorical variables and as mean \pm standard deviations for continuous variables. $p$-values were tested by one-way analysis of variance, followed by a post-hoc Tukey comparison for continuous variables and $\chi^{2}$ test for categorical variables. $* p<0.05$ vs. low education group. EQ-5D: EuroQoL 5-dimensions, EQ-VAS: EuroQoL-visual analog scale, HL: hearing loss, Low: low education, Middle: middle education, High: high education

$(9.0 \%)$ in the low education group and $37(4.7 \%)$ in the nonlow education group ( $p=0.001)$. On univariate logistic regression analysis, the low education group had an odds ratio of 1.17 (95\% CI: $0.92-1.50 ; p=0.195$ ) for any tinnitus and 1.98 (95\% CI: $1.31-2.99 ; p=0.001$ ) for moderate to severe tinnitus compared to non-low education group. Multivariate logistic regression analysis, adjusted for occupation, showed that the low education group had an odds ratio of 1.17 (95\% CI: 0.91$1.49 ; p=0.217)$ for any tinnitus and 1.97 (95\% CI: 1.30-2.97; $p=0.001$ ) for moderate to severe tinnitus compared to the nonlow education group.

QoL scales according to education level are shown in Supplementary Table 2. Participants who had problems with mobility, usual activities, or experienced pain/discomfort were higher in the low education group compared to the non-low education group. On multivariate analysis adjusted for occupation and severity of tinnitus, EQ-5D index in the low and the non-low education groups were $0.91 \pm 0.01$ (mean $\pm \mathrm{SE}$ ) and $0.94 \pm 0.01(p=0.009)$. EQ-VAS in the low and the nonlow education groups were $69.7 \pm 1.3($ mean \pm SE) and $74.2 \pm$
1.4 ( $p=0.024)$ respectively. There were significant differences in EQ-5D index and EQ-VAS between the low and nonlow education groups on univariate and multivariate analysis.

\section{Discussion}

Our study revealed that a low education level is associated with moderate to severe tinnitus among the Korean adult population. For subjects with tinnitus, low education level was associated with poor QoL scores. Subgroup analysis of participants with HL was also similar to those including all participants.

First, we evaluated the association between education level and tinnitus in the Korean adult population. Although the exact mechanisms behind the association between education level and tinnitus are unknown, some researchers suggest that low education levels lead to less effective coping strategies, diminished self-esteem, with lower cognitive and control capacities in a given situation. ${ }^{7,11}$ These may, in turn, lead to a high prevalence of tinnitus in subjects who have a low level of education. However, there are other studies that failed to 
identify an association between tinnitus and the level of education. Shargorodsky et al. ${ }^{1)}$ on analysis of the NHANES data showed that the prevalence of tinnitus was generally higher with a low level of education; however, variable adjusted odds ratios did not reveal statistical significance. Two studies among the Japanese population evaluated the prevalence of and risk factors for tinnitus. ${ }^{2,12}$ They also analyzed education level as a potential risk factor; however, statistical significance was only in one study. ${ }^{2)}$ The positive result was shown solely among men, with multivariate analysis adjusted only for age. A study among the Korean population showed a positive association between education level and tinnitus on univariate analysis; however, the association was not significant on multivariate analysis. ${ }^{3)}$ These negative studies results used any degree of tinnitus as a dependent variable. ${ }^{1-3,12)}$ However, other studies have shown a positive association between education level and severity of tinnitus. ${ }^{7,13)}$ These conflicting findings (positive association between education level and severity of tinnitus and negative association between education level and prevalence of tinnitus) suggest that education level is not associated with the prevalence of any tinnitus, but the association may hold true for high grade tinnitus. Our study showed that education level was not associated with any tinnitus; however, there was a positive association between education level and moderate to severe tinnitus as observed on univariate, multivariate, and propensity analyses.

Second, we evaluated the effect of education level on QoL scales in participants with tinnitus. Many clinicians accept the effect of tinnitus on QoL; the impact of tinnitus on QoL varies from no effect to development of suicidal ideation. ${ }^{14)}$ However, there are few studies that have addressed the influence of tinnitus on QoL in participants with tinnitus, except in subjects with HL and severe tinnitus. ${ }^{5,6)}$ Although there are few studies regarding the association between education level and QoL in participants with tinnitus, some factors may influence the relationship between these variables.

A meta-analysis of eight randomized controlled trials showed a favorable effect of psychological and cognitive behavioral therapy on QoL in subjects with tinnitus. ${ }^{15)}$ A clinical practice guideline based on these results recommends psychological therapies rather than use of medication for tinnitus. ${ }^{8)}$ Effectiveness of psychological therapy in tinnitus may constitute indirect evidence that supports the association between education level and QoL in subjects with tinnitus. Psychological therapy such as cognitive behavioral therapy is based on cognitive restructuring of thoughts and exposure to inducing fac- tors through education and/or counseling. ${ }^{15)}$ Participants with a high level of education may be more amenable to such therapy than those with a low level of education. In addition, in our study, participants with a high level of education had higher income and less labor-intensive occupation than those with a low level of education. In Korea, most hospitals are in metropolitan cities, while participants such as farmers or fishermen reside far away from cities. ${ }^{16)}$ The type of occupation, poor income and a low level of education may be associated with less access to health case. Although our data did not include the presence, frequency, or modality of treatment for tinnitus and accessibility for hospital, future studies including effect of psychologic therapy or hospital accessibility according to the level of education may be interesting.

Our study has several strengths. First, our cohort included clinical data on evaluation of the tympanic membrane and the presence of otitis media; tympanic membrane abnormalities or a history of otitis media could influence of development of tinnitus and affect QoL. We were able to exclude these factors by performing otological examination on all participants. Second, we evaluated the association between variables in detail using appropriate statistical methods. In our cohort, baseline characteristics were different between groups, which may have confounded analysis. However, we performed multivariate, subgroup and propensity matched analysis to adjust for the difference in baseline characteristics. In addition, we performed subgroup analyses according to the presence of HL, which is closely associated with the prevalence or severity of tinnitus. Third, we adjusted for potential confounding factors using multivariate analyses and used for propensity matching. Thorough evaluation for comorbidities was another strength of our study, although the association between comorbidities such as DM, HTN, CVA, CAD, osteoarthritis, rheumatoid arthritis, dyslipidemia, or depression and tinnitus are controversia. $^{10,17-19)}$ DM, HTN, CVA, CAD, and the drugs used to treat these conditions, including aspirin, may also influence. ${ }^{1,17,18)}$ In addition, osteoarthritis and rheumatoid arthritis could influence tinnitus by an autoimmune mechanism or by medication including non-steroidal anti-inflammatory drugs. ${ }^{17-19)}$

Our study has some limitations. First, our study design was retrospective cross-sectional. We did not evaluate a cause and effect-relationship between education level and outcomes. Second, exclusion rate was high, with 9977 (76.0\%) participants being excluded mainly due to incomplete data. The high exclusion rate may have led to a selection bias. Third, we did not evaluate the presence and grade of tinnitus using a tinni- 
tus specific instrument such as Tinnitus Handicap Inventory. Fourth, we did not evaluate the impact of drug treatment or access to health care.

In conclusion, our study showed that low education level was associated with moderate to severe tinnitus in the Korean adult population. Participants with tinnitus and a low level of education had poorer QoL than those with tinnitus and a high level of education. Prospective studies including additional data using a tinnitus-specific instrument and details of various treatment modalities are needed to assess a potential cause and effect-relationship between variables that may impact the prevalence and severity of tinnitus.

\section{Acknowledgments}

This work was funded by the grants of the National Research Foundation of Korea Grant 2018R1C1B6007775.

\section{ORCID}

Kyu-Yup Lee

https://orcid.org/0000-0001-7170-4847

\section{REFERENCES}

1) Shargorodsky J, Curhan GC, Farwell WR. Prevalence and characteristics of tinnitus among US adults. Am J Med 2010;123 (8):711-8

2) Fujii K, Nagata C, Nakamura K, Kawachi T, Takatsuka N, Oba $S$, et al. Prevalence of tinnitus in community-dwelling Japanese adults. J Epidemiol 2011;21(4):299-304.

3) Kim HJ, Lee HJ, An SY, Sim S, Park B, Kim SW, et al. Analysis of the prevalence and associated risk factors of tinnitus in adults. PLoS One 2015;10(5):e0127578.

4) Baguley D, McFerran D, Hall D. Tinnitus. Lancet 2013;382(9904): 1600-7.

5) Joo YH, Han KD, Park KH. Association of hearing loss and tinnitus with health-related quality of life: The Korea National Health and Nutrition Examination Survey. PLoS One 2015;10(6):e0131247.

6) Zeman F, Koller M, Langguth B, Landgrebe M; Tinnitus Research Initiative database study group. Which tinnitus-related aspects are relevant for quality of life and depression: Results from a large international multicentre sample. Health Qual Life Outcomes 2014; 12:7.
7) Unterrainer J, Greimel KV, Leibetseder M. Are demographic and socioeconomic factors predictive for perceived tinnitus impairment? Int Tinnitus J 2001;7(2):109-11.

8) Tunkel DE, Bauer CA, Sun GH, Rosenfeld RM, Chandrasekhar SS, Cunningham ER Jr, et al. Clinical practice guideline: Tinnitus executive summary. Otolaryngol Head Neck Surg 2014;151(4):53341.

9) The Korea Centers for Disease Control and Prevention. Alcohol. [cited 2017 Sep 26]. Available from: URL: http://health.mw.go.kr/ HealthInfoArea/HealthInfo/View.do?id $x=5800$ \&subId $x=4 \&$ searchCate $=\&$ searchType $=\&$ search $\mathrm{Key}=\&$ page $\mathrm{No}=\&$ category $=1$ $\&$ category_code $=301301306 \&$ dept $=3 \&$ sortType $=$ date $\&$ page $=1 \& \mathrm{~s}$ earchField $=$ titleAndSummary\&searchWord $=$.

10) EuroQoL Research Foundation. EQ-5D. [cited 2017 Sep 26]. Available from: URL: http://www.euroqol.org.

11) Lee YK, Nam HS, Chuang LH, Kim KY, Yang HK, Kwon IS, et al. South Korean time trade-off values for EQ-5D health states: Modeling with observed values for 101 health states. Value Health 2009;12(8):1187-93.

12) Michikawa T, Nishiwaki Y, Kikuchi Y, Saito H, Mizutari K, Okamoto M, et al. Prevalence and factors associated with tinnitus: A community-based study of Japanese elders. J Epidemiol 2010;20 (4):271-6.

13) Hoekstra CE, Wesdorp FM, van Zanten GA. Socio-demographic, health, and tinnitus related variables affecting tinnitus severity. Ear Hear 2014;35(5):544-54.

14) Turner O, Windfuhr K, Kapur N. Suicide in deaf populations: A literature review. Ann Gen Psychiatry 2007;6:26.

15) Martinez-Devesa P, Perera R, Theodoulou M, Waddell A. Cognitive behavioural therapy for tinnitus. Cochrane Database Syst Rev 2010;8(9):CD005233.

16) Health Insurance Review \& Assessment Service. Healthcare Bigdata Hub. [cited 2017 Sep 26]. Available from: URL: http:// opendata.hira.or.kr/op/opc/yadmOpCloPrsnt.do?searchType $=\&$ dateTyp $\mathrm{e}=\&$ frYear $=2017 \&$ frMonth $=06 \&$ ykihoPlcTpCd=\&ykihoPl $\mathrm{cTpCdNm}=\& \mathrm{clCd}=01 \&$ shwSbjtC $\mathrm{d}=\#$ none.

17) Perry B, Gantz B. Medical and surgical evaluation and management of tinnitus. In: Tyler RS, editor. Tinnitus Handbook. San Diego, CA: Singular Publishing Group;2000. p.221-41.

18) Nondahl DM, Cruickshanks KJ, Huang GH, Klein BE, Klein R, Nieto FJ, et al. Tinnitus and its risk factors in the Beaver Dam Offspring Study. Int J Audiol 2011;50(5):313-20.

19) Nondahl DM, Cruickshanks KJ, Wiley TL, Klein BE, Klein R, Chappell R, et al. The ten-year incidence of tinnitus among older adults. Int J Audiol 2010;49(8):580-5. 


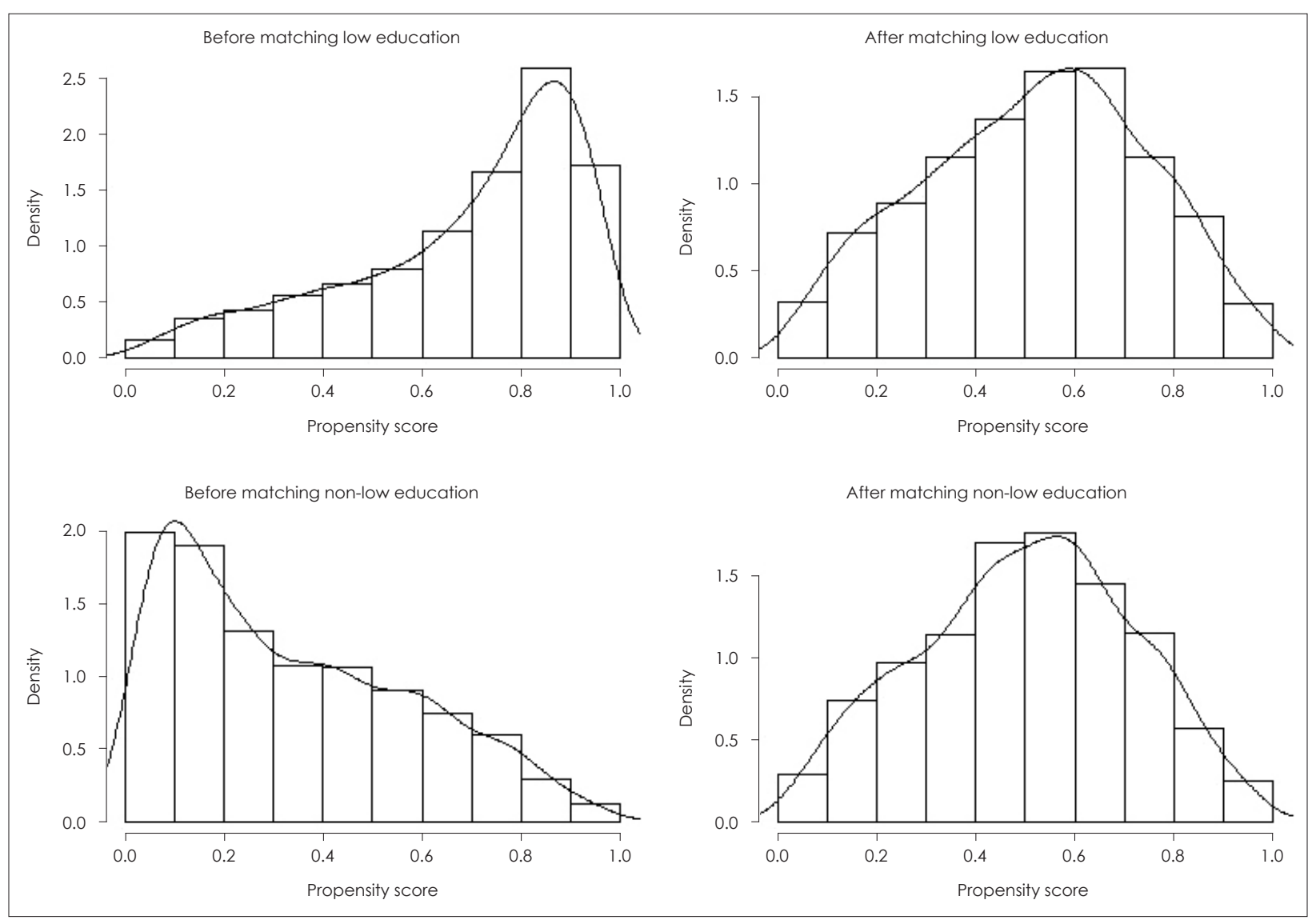

Supplementary Fig. 1. Distribution of propensity scores before and after matching. The distribution of propensity scores before matching differed between the low education and non-low education groups, but there was no difference after matching. 
Supplementary Table 1. Participants' characteristics according to education level after propensity score matching

\begin{tabular}{|c|c|c|c|}
\hline Variables & Low education group $(n=782)$ & Non-low education group $(n=782)$ & p-value \\
\hline Age (years) & $57.4 \pm 7.9$ & $57.0 \pm 9.8$ & 0.383 \\
\hline Sex (men, \%) & $362(46.3)$ & $373(47.7)$ & 0.577 \\
\hline Body mass index $\left(\mathrm{kg} / \mathrm{m}^{2}\right)$ & $24.0 \pm 3.1$ & $23.9 \pm 3.1$ & 0.752 \\
\hline Diabetes mellitus (\%) & $112(14.3)$ & $111(14.2)$ & 0.942 \\
\hline Hypertension (\%) & $323(41.3)$ & $304(38.9)$ & 0.327 \\
\hline Smoking habitus (\%) & & & 0.598 \\
\hline Non-smoker & $449(57.4)$ & $431(55.1)$ & \\
\hline Ex-smoker & $190(24.3)$ & $206(26.3)$ & \\
\hline Current smoker & $143(18.3)$ & $145(18.5)$ & \\
\hline Alcohol intake (\%) & & & 0.079 \\
\hline Abstinence & $244(31.2)$ & $227(29.0)$ & \\
\hline Moderate intake & $486(62.1)$ & $520(66.5)$ & \\
\hline Heavy intake & $52(6.6)$ & $35(4.5)$ & \\
\hline Coronary artery disease (\%) & $36(4.6)$ & $33(4.2)$ & 0.712 \\
\hline Cerebrovascular accident (\%) & $18(2.3)$ & $15(1.9)$ & 0.598 \\
\hline Average hearing threshold (dB) & $20.9 \pm 12.9$ & $20.2 \pm 13.2$ & 0.291 \\
\hline Household income (1000 won/month) & $355.5 \pm 792.5$ & $416.0 \pm 759.8$ & 0.123 \\
\hline Exposure to occupational noise (\%) & $120(15.3)$ & $119(15.2)$ & 0.944 \\
\hline Exposure to explosive noise (\%) & $164(21.0)$ & $164(21.0)$ & 1.000 \\
\hline Occupation & & & $<0.001$ \\
\hline Non-labor intensive (\%) & $244(31.2)$ & $353(45.1)$ & \\
\hline Labor intensive (\%) & $272(34.8)$ & $106(13.6)$ & \\
\hline Non-occupation (\%) & $266(34.0)$ & $323(41.3)$ & \\
\hline Osteoarthritis (\%) & $86(11.0)$ & $83(10.6)$ & 0.807 \\
\hline Rheumatoid arthritis (\%) & $19(2.4)$ & $18(2.3)$ & 0.868 \\
\hline Dyslipidemia (\%) & $118(15.1)$ & $116(14.8)$ & 0.887 \\
\hline Depression (\%) & $32(4.1)$ & $37(4.7)$ & 0.538 \\
\hline Stress (\%) & & & 0.469 \\
\hline No & $135(17.3)$ & $128(16.4)$ & \\
\hline Some stress & $480(61.4)$ & $476(60.9)$ & \\
\hline Moderate stress & $133(17.0)$ & $152(19.4)$ & \\
\hline Severe stress & $34(4.3)$ & $26(3.3)$ & \\
\hline
\end{tabular}

The data are expressed as counts (percentages) for categorical variables and as mean \pm standard deviations for continuous variables. $p$-values were tested by t-test and $\chi^{2}$ test for categorical variables 
Supplementary Table 2. Quality of life scales according to education level in participants with tinnitus using propensity score matched cohort

\begin{tabular}{lccc}
\hline & Low & Non-low & p-value \\
\hline Mobility (\%) & $40(22.6)$ & $20(12.8)$ & 0.021 \\
Self-care (\%) & $8(4.5)$ & $3(1.9)$ & 0.186 \\
Usual activities (\%) & $31(17.5)$ & $12(7.7)$ & 0.008 \\
Pain/discomfort (\%) & $60(33.9)$ & $35(22.4)$ & 0.021 \\
Anxiety/depression (\%) & $31(17.5)$ & $25(16.0)$ & 0.717 \\
EQ-5D index & $0.91 \pm 0.13$ & $0.95 \pm 0.08$ & 0.002 \\
EQ-VAS & $69.4 \pm 18.1$ & $74.5 \pm 17.5$ & 0.010
\end{tabular}

The data are expressed as counts (percentages) for categorical variables and as mean \pm standard deviations for continuous variables. $p$-values were tested by t-test and $\chi^{2}$ test for categorical variables. EQ-5D: EuroQoL 5-dimensions, EQ-VAS: EuroQoLvisual analog scale, Low: low education, Non-low: non-low education 